Journal of

Accident and

Emergency

Medicine 1994

11, 259-260

\title{
Congestive cardiac failure and aortic dissection in a young man with Marfan's syndrome
}

\author{
S. AGRAWAL, S. LONGFIELD \& G.GEORGE
}

Accident and Emergency Department, Horton General Hospital NHS Trust, Banbury, Oxon

\section{INTRODUCTION}

Marfan's syndrome is inherited as an autosomal dominant trait with variable penetrance. It affects the skeleton, eyes and cardiovascular system. There is disproportionate longitudinal bone growth resulting in long limbs, arachnodactyly, dolichocephaly, high-arched palate and a long, narrow face. Recurrent joint dislocations and kyphoscoliosis are seen. In the eye, lens dislocation is the most common abnormality but myopia and retinal detachment are also described. The cardiovascular abnormalities deserve special attention because they pose the most serious threat to life. Aneurysmal dilation of the aorta is well-recognized, most commonly occurring at the root of the aorta with natural progression to dissection and rupture. Cardiac failure may be precipitated by aortic or mitral valve prolapse, and rarely by myocardial infarction. ${ }^{1}$ Annual measurement of aortic diameter has been recommended in young people despite a low prevalence of serious cardiovascular complications. ${ }^{2}$ Other cardiovascular complications have been described for example, atrial fibrillation and interatrial septal aneurysm, ${ }^{3}$ and dissection of the internal carotid artery. ${ }^{4}$ It appears that any major artery can become ectatic.

Key words: aortic dissection, cardiac failure, Marfan's syndrome

\section{CASE REPORT}

A 22-year-old male Yugoslav refugee, recently arrived in the UK, presented to the accident and emergency (A\&E) department. His English was poor. He gave a 3-month history of breathlessness, much worse over the previous 3 days. He had been unable to sleep because of shortness of breath. He felt tired and weak and had lost weight. He described a vague, left-sided, pleuritic chest pain. There was no known family history of Marfan's syndrome. He smoked 20 cigarettes per day.

On examination, he looked pale and unwell. He was clammy and dyspnoeic. The pulse was 110 beats $\min ^{-1}$, the blood pressure was $137 / 60$ in both arms. The apex beat was displaced and visibly hyperdynamic. There was an apical thrill and left parasternal heave. He had a gallop rhythm. There were systolic and diastolic murmurs which were difficult to characterize. The jugular venous pressure was raised but there was no hepatomegaly or ankle swelling. There were coarse crepitations at the right base and reduced air entry at the left base. He was noted to be unusually thin and tall but other features

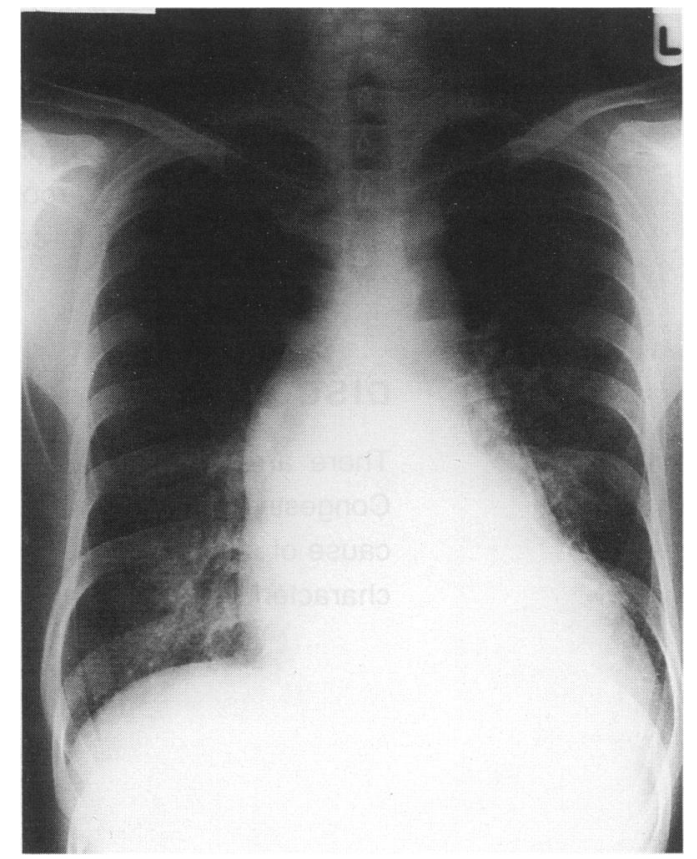

Fig. 1. Chest radiograph in a patient with Marfan's syndrome showing cardiomegaly, unfolded aorta, widened mediastinum, and the features of left ventricular failure. 
Cardiac failure and aortic dissection with Marfan's Syndrome

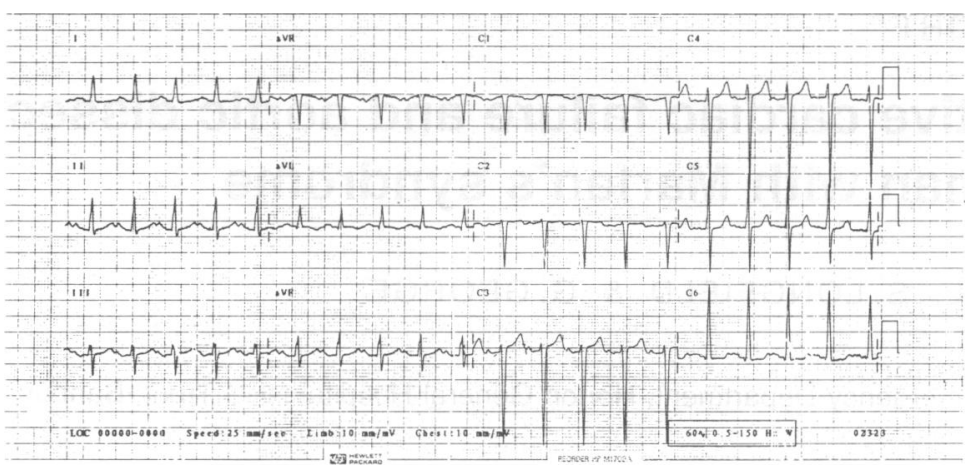

Fig. 2. ECG showing left ventricular hypertrophy.

of Marfan's syndrome were not recognized at this stage. He was apyrexial.

Chest radiograph showed gross cardiomegaly, a widened mediastinum with unfolding of the aorta, shadowing at the right base, upper lobe diversion, Kerley $B$ lines, and fluid in the transverse fissure (Fig. 1). The ECG showed a sinus rhythm with left ventricular hypertrophy (Fig. 2).

A diagnosis was made of left ventricular failure secondary to mixed valvular disease. In addition, aneurysmal dilation of the aorta was noted. The valvular disease was at first thought to be either congenital or rheumatic. However, features of Marfan's syndrome were subsequently recognized and the aetiology of the valvular and aortic disease was understood. The possibility of associated infection was considered, including subacute bacterial endocarditis. He was treated symptomatically with oxygen and diuretics.

Urgent echocardiography showed aortic regurgitation and aortic root dissection. The mitral valve was normal. He urgently underwent replacement of his aortic valve, aortic root and descending aorta. After some initial complications, he made a good recovery.

\section{DISCUSSION}

There are lessons to be learned from this case. Congestive cardiac failure is a very uncommon cause of shortness of breath in a young adult. The characteristics of Marfan's syndrome were initially missed in a patient who was incapacitated by breathlessness and who was acutely unwell. The aortic dissection was unusual in that it appeared to be relatively painless although it is possible that his pain was underestimated because of difficulties in communication. In consequence, the aortic aneurysm was not suspected until the widened mediastinum was seen on chest radiograph. The valvular incompetence and aortic dissection required urgent surgical correction and prompt diagnosis was crucial to a successful outcome.

\section{ACKNOWLEDGEMENTS}

We should like to thank the Radiography department, Dr J. Privett and Dr P. Roche for assistance.

\section{REFERENCES}

1. Skribnik, E. \& Oteva, E.A. (1991) Rare types of myocardial infarction in young patients. Klinicheskaia Meditsina (Moskva) 69, 32-35.

2. el-Habbab, M.H. (1992) Cardiovascular manifestations of Marfan's syndrome in the young. American Heart Journal 123, 752-757.

3. Cronin, C.C. \& Harris, A.M. (1992) Atrial fibrillation and interatrial septal aneurysm in a patient with Marfan's syndrome. International Journal of Cardiology 34, 115-117.

4. Schulze, H.E., Ebner, A. \& Besinger, U.A. (1992) Report of dissection of the internal carotid artery in three cases. Neurosurgery Review 15, 61-64. 